\title{
Ontologie et anthropologie
}

Dix ans de controverse (Brésil, France, États-Unis)

Onthology and anthropology: ten years of controversy (Brazil, France, United States)

\section{Erwan Dianteill}

\section{OpenEdition \\ Journals}

Édition électronique

URL : https://journals.openedition.org/ress/3314

DOI : $10.4000 /$ ress.3314

ISBN : 1663-4446

ISSN : 1663-4446

Éditeur

Librairie Droz

\section{Édition imprimée}

Date de publication : 15 novembre 2015

Pagination : 119-144

ISSN : 0048-8046

\section{Référence électronique}

Erwan Dianteill, « Ontologie et anthropologie », Revue européenne des sciences sociales [En ligne], 53-2 I 2015, mis en ligne le 15 novembre 2018, consulté le 21 septembre 2021. URL : http:// journals.openedition.org/ress/3314; DOI : https://doi.org/10.4000/ress.3314 


\title{
ONTOLOGIE ET ANTHROPOLOGIE \\ DIX ANS DE CONTROVERSE (BRÉSIL, FRANCE, ÉTATS-UNIS)
}

ERWAN DIANTEILL

Université Paris Descartes, CANTHEL - Institut Universitaire de France erwan.dianteill@parisdescartes.fr

\begin{abstract}
Résumé. Le concept d'ontologie est aujourd'hui objet de vifs débats en anthropologie culturelle et sociale. Alors qu'il s'agit d'un concept éminemment philosophique, il est passé dans le vocabulaire des sciences sociales depuis une vingtaine d'années. Le moment clef de cette incorporation est la publication de l'ouvrage Par-delà nature et culture (2004) de Philippe Descola, traduit en anglais en 2013. Mais dès avant la publication de l'ouvrage, le dialogue que Descola avait eu avec Eduardo Viveiros de Castro avait contribué à la formation de son modèle des quatre ontologies. Comme nous le verrons, la réception contrastée du livre en France s'y explique par la diversité de la discipline anthropologique. Le débat s'est ensuite prolongé aux États-Unis avec la publication du livre dans ce pays. L'anthropologie des ontologies a-t-elle passé avec succès le test de résistance de la critique internationale? Tel est l'objet de cet article.
\end{abstract}

Mots-clés: anthropologie, culture, matérialisme, nature, ontologie, perspectivisme, Philippe Descola, postmodernisme, structuralisme.

\begin{abstract}
The concept of ontology is currently hotly debated in cultural and social anthropology. While it was a philosophical concept, it has been incorporated into the vocabulary of the social sciences for twenty years. The key moment of this incorporation is the publication of the book Beyond nature and culture (2004) by Philippe Descola, translated into English in 2013. But even before the publication of the book, the dialogue that Descola has had with Eduardo Viveiros de Castro had helped him to form his model of the four ontologies. As it is explained, the mixed reception of the book in France is the product of the diversity of the anthropological discipline. Then the debate was extended to the United States with the book's publication in that country. Has the anthropology of ontologies passed successfully this stress test? This is the purpose of this article.
\end{abstract}

Keywords: anthropology, culture, materialism, nature, ontology, perspectivism, Philippe Descola, postmodernism, structuralism. 
INTRODUCTION

Avant les années 1990, le concept d'ontologie appartenait exclusivement au vocabulaire philosophique, et semblait étranger aux sciences sociales'. En tant que «science de l'être», l'ontologie a une longue histoire, qui trouve son origine chez Platon (Le Sophiste), peut-être même chez les présocratiques, avec Parménide (Le Poème). Si la réflexion sur l'être date de la Grèce antique, et s'est épanouie avec la scolastique du Moyen Âge (Saint Anselme, Saint-Thomas), le mot est créé en latin en 1606 par Jacob Lorhard (I56I-1609), philosophe méconnu, recteur au Gymnasium de Saint Gallen (Suisse), puis il est repris par Rudolf Göckel (I547-1628), professeur de logique à l'université de Marburg. Il connaît ensuite une grande fortune à l'époque moderne et contemporaine, qui culmine au $\mathrm{Xx}^{\mathrm{e}}$ siècle avec la phénoménologie de Husserl et l'existentialisme de Heidegger et Sartre².

On chercherait en vain en revanche, même chez les théoriciens des sciences sociales les plus portés à l’abstraction, une reprise de la tentative de «science de l'être » des philosophes. Tout se passe au contraire comme si les fondateurs de la sociologie et de l'anthropologie culturelle avaient souhaité éviter de s'engager sur le chemin de la métaphysique. Or, l'ontologie en est un synonyme, dès que le mot apparaît dans le vocabulaire de la philosophie. Les sciences sociales, si elles souhaitent faire ouvre de science, doivent se maintenir dans l'étude des faits constatables, voilà leur postulat. L'étude des choses en soi, de l'être, de la substance, voilà un projet non seulement étranger aux sciences de l'homme, mais même considéré comme pernicieux par les trois «pères» des sciences sociales: Marx, Durkheim et Weber convergent sur ce point, malgré toutes leurs différences épistémologiques.

I Le point de départ de cet article est une invitation à débattre avec Philippe Descola et Richard Pottier, débat organisé par Yves-Charles Zarka le 8 mars 2014 en Sorbonne, sur le thème de l'humanisme dans la diversité culturelle; on trouvera le point de vue de RichardPottier exposé plus loin, le mien en conclusion.

2 II n'est pas question de faire ici l'histoire de l'ontologie en philosophie, ni même celle du mot «ontologie». On consultera sur ces sujets, avec grand profit, le site Theory and history of ontology animé par Raul Corazzon, qui brille par son érudition: <http://www.ontology.co>. Voir en particulier l'article bibliographique (Corazzon, 201I). 
Or, avec la publication de Par-delà nature et culture (2005), Philippe Descola ${ }^{3}$ a replacé l'ontologie (ou plutôt les ontologies, nous allons le voir) au cœur du projet de connaissance de l'anthropologie culturelle et sociale. C'est une inflexion significative pour la discipline (même si la réflexion ontologique date des années 1990), car les propositions de Descola conduisent à s'interroger sur ce qui, anthropologiquement, est réel. Alors que jusqualors, c'est plutôt la question du symbolisme, de la représentation et du sens qui organisait le débat en anthropologie fondamentale, cela dans le sillage de La Pensée sauvage de Lévi-Strauss (1962), le problème de l'être devient central. Lorsque les Achuars disent que les jaguars sont leurs beaux-frères, doit-on considérer que cet énoncé est une façon de parler, une figure de style, une sorte de métaphore et de jeu de langage, ou bien que les Achars pensent ce qu'ils disent? Ce langage exprime-t-il leur certitude de ce qu'est un jaguar, ou sont-ils conscients que la réalité animale n'est en fait nullement familière, même si le vocabulaire utilisé pour en parler appartient au registre de la parenté ? Il y a évidemment une différence essentielle à considérer les jaguars comme des beaux-frères, ou à les prendre effectivement pour des beaux-frères (ce qui pourrait s'avérer périlleux).

Plus généralement, le travail de Descola sur la question de l'être en anthropologie ouvre la voie à toute une série d'interrogations fondamentales. Doit-on considérer que la conception de la réalité est variable selon les cultures? Le nombre d'ontologies est-il illimité? Toutes les ontologies se valent-elles du point de vue de la vérité? Quel est le critère nous permettant de décider que l'une vaut plus que l'autre? En dix ans, de multiples lectures critiques de Par-delà nature et culture ont été publiées, et l'intérêt pour ce livre a été relancé récemment par une édition en langue anglaise préfacée par Marshall Sahlins (20I3). Ces lectures adoptent des points de vue variés sur l'ouvrage, et éclairent les forces et les faiblesses du concept d'ontologie en anthropologie culturelle et sociale. La thèse de Descola a-t-elle passé avec succès ce «test de résistance» de la critique internationale ${ }^{4}$.

3 Philippe Descola est professeur au Collège de France, où il est titulaire de la chaire d'anthropologie de la nature.

4 Le «stress test» est une technique visant à évaluer la stabilité d'un dispositif technique dans des conditions de fonctionnement sévères ou extrêmes; il s'agit parfois de tests expérimen- 
Trois moments scandent la réception de l'œuvre. Le premier prend paradoxalement sa source avant sa publication en 2005 : il s'agit du débat entre Eduardo Viveiros de Castro et Descola qui débute dans les années 1990 et se poursuit dans les années 2000. Le deuxième est celui de sa réception française avec un ensemble de recensions publiées en 2006. Enfin, un troisième temps correspond à la traduction américaine et sa réception: un symposium publié par la revue HAU : Journal of Ethnographic Theory (20I4) nous en donnera une première image.

\section{LE DÉBAT VIVEIROS DE CASTRO VS DESCOLA}

Eduardo Viveiros de Castro, professeur au musée national de Rio de Janeiro, et Philippe Descola sont des anthropologues spécialistes de peuples amérindiens relativement proches par leur vision du monde. Le premier a réalisé l'ethnographie des Yawalapíti du Haut Xingu, dans l'État brésilien du Moto Grosso, le second celles des Achuars (plus connus sous le nom de Jivaros) qui vivent en Amazonie, en Équateur. Dans les années 1990, le dialogue entre eux a conduit Viveiros de Castro à formuler le concept de «perspectivisme », tandis que Descola reprenait celui d' «animisme », utilisé dès le XIX siècle par Edward B. Tylor, afin de rendre compte de la cosmologie des Amérindiens. Pour comprendre leurs différends, il faut d’abord présenter rapidement la théorie de Viveiros de Castro.

Le perspectivisme a trouvé une première expression dans un article intitulé «Les pronoms cosmologiques et le perspectivisme amérindien » (Viveiros de Castro, 1996, traduit par nous ${ }^{5}$. Comment, selon les Amérindiens, les humains, les animaux et les esprits se voient-ils les uns les autres? Car le monde est pour ces gens peuplé de personnes qui peuvent être des hommes, des animaux ou des esprits, et chacun de ces sujets ont sur le monde un point de vue spécifique. Or, cette généralisation amérindienne du statut de sujet aux animaux, aux esprits et peut-être même à certaines choses est incompatible avec la conception euro-

taux (matériaux et machines), ou de simulations (institutions bancaires). Je propose d'adapter cette idée aux sciences sociales en considérant qu'un corpus de recensions et de notes critiques, sur le long terme, constitue le test de résistance d'un ouvrage dans nos disciplines.

5 Toutes les citations qui suivent de Viveiros de Castro sont traduites par nous. 
péenne d'une différence radicale entre Nature et Culture, telle qu'elle est développée dans l'ethnologie «classique». Il faut donc en faire la critique :

Une telle critique, dans le cas présent, exige la redistribution des prédicats inclus dans les deux séries paradigmatiques qui s'opposent traditionnellement sous les étiquettes de Nature et de Culture : l'universel et le particulier, l'objectif et le subjectif, le physique et le moral, le fait et la valeur, le donné et le construit, la nécessité et la spontanéité, l'immanence et la transcendance, le corps et l'esprit, l'animalité et l'humanité, et bien d'autres encore (ibid., p. II5-116).

Tout comme Tim Ingold avant lui (1991, p. 356), Viveiros de Castro définit la cosmologie occidentale comme universalité de la Nature et multiplicité des Cultures. Dans la cosmovision moderne européenne, les éléments naturels sont homogènes, présents partout dans le monde, tandis que les constructions symboliques, les langues, les croyances, les rituels, les mœurs des humains en général sont extrêmement variables et hétérogènes. En revanche, les Amérindiens ont une toute autre cosmovision:

Notre cosmologie postule une continuité physique et une discontinuité métaphysique (c'est-à-dire, «surnaturelle», si l'on utilise une racine latine grecque plutôt que latine) entre les humains et les animaux, la première faisant de l'homme l'objet des sciences naturelles, la seconde, des sciences de la culture. L'esprit est le grand différenciateur occidental : c'est ce qui nous place au-dessus des animaux et de la matière en général, ce qui nous singularise face à nos semblables, ce qui distingue les cultures. Le corps, au contraire, est le grand intégrateur: il nous connecte au reste des vivants, tous unis par un substrat universel (ou ADN, chimie du carbone, etc.) qui à son tour, renvoie à la nature fondamentale de tous les corps matériels. En revanche, les Amérindiens postulent une continuité métaphysique et une discontinuité physique entre les êtres du cosmos, la première conduisant à l'animisme, la seconde au perspectivisme: l'esprit (qui n'est pas ici une substance immatérielle, mais une forme réflexive) est ce qui intègre; le corps (qui n'est pas substance matérielle, mais affection active) est ce qui différencie (Viveiros de Castro, 1996., p. I29).

Les lignes qui précèdent sont très proches de ce que Descola appelle «naturalisme» d'un côté et «animisme» de l'autre. En 1986, ce dernier écrivait déjà à propos de l'animisme amérindien: «le référentiel commun à tous les êtres de la nature n'est pas l'homme en tant qu'espèce, mais l'humain en tant 
que condition » (1986, p. I20). Pourtant, il existe plusieurs différences notables entre les propositions théoriques de Viveiros de Castro et de Descola. Ainsi, le premier ne se contente pas d'avancer que pour les Amérindiens, hommes, esprits et choses ont une âme, c'est-à-dire que ce sont des sujets; il combine cette idée avec celle que les êtres vivants, de par leurs différentes constitutions corporelles qui ne se réduisent pas à un agencement organique, mais se constituent comme une façon d'être, un habitus, sont porteurs de différents points de vue: «Entre la subjectivité formelle des âmes et la matérialité substantielle des organismes, il existe cette ossature qu'est le corps comme faisceau d'affections et de capacités, et qui est à l'origine des perspectives. » (ibid., p. I28). Si l'on s'en tient à une théorie de l'animisme, on manque donc ce qui en est inséparable, à savoir la compréhension du point de vue des sujets non-humains. Or, pour Viveiros de Castro, les animaux prédateurs perçoivent les humains comme des proies animales, tandis que les animaux-proies voient les humains comme des animaux prédateurs (car ces derniers les tuent et les mangent), et tous ces animaux, quels qu'ils soient, se conçoivent comme des humains.

Il semble que nous soyons ici face à un relativisme radical, chaque espèce ayant une visée différente sur le monde. Pourtant, Viveiros de Castro rejette absolument l'idée que le perspectivisme serait un relativisme, au sens où chaque vision du monde serait relative à une subjectivité particulière. Les différents points de vue n'attestent en fait en rien de l'existence d'un multiculturalisme, au sens où chaque espèce naturelle aurait une vue différente sur un même monde : «Le perspectivisme est un multinaturalisme, car une perspective n'est pas une représentation» (1996, p. I28). Ce qui varie, ce n’est pas la subjectivité, c'est le monde. Les animaux voient les choses comme nous, avec les mêmes valeurs et les mêmes catégories, mais ils ne voient pas la même chose. Ainsi, ce qui pour nous est du sang est pour le jaguar de la bière de manioc (cauim). Le monde objectif n'est donc pas le même pour l'animal que pour l'homme, bien que la forme de sa subjectivité soit la même que la nôtre. C'est pourquoi le perspectivisme amérindien affirme l'universalité de la culture et la relativité de la nature (si tant est que ces concepts aient encore un sens dans 
une telle cosmovision), et non l'inverse, qui caractérise le relativisme culturel dans une vision du monde de type naturaliste.

Pourtant, si Descola et Viveiros de Castro semblaient partager une même conception de l'animisme au début des années 1990, le désaccord est manifeste dès 1998, année où l'anthropologue brésilien enseigne à l'École pratique des hautes études ( $5^{\mathrm{e}}$ section). Il expose, lors de sa deuxième conférence, ce qui le sépare de Descola (Viveiros De Castro, 1998, p. 106). Ce n’est pas l'attribution de la qualité de sujet à des êtres qui passent pour ne pas avoir d'intentionnalité en Occident qui fait le départ entre les deux théories, mais bien le degré de réalisme qu'il faut accorder à cette hypothèse. Selon le Brésilien, Descola considère que lorsque les Amérindiens disent que les animaux sont des hommes, il s'agit d'une projection du monde social sur le monde naturel, présupposant qu'il y a un mode social et intersubjectif réel (celui des hommes), et que la socialité des jaguars ou des tapirs n’est en revanche que métaphorique. En s'inspirant de Benveniste et de son analyse des pronoms personnels («je» et «tu» sont les seuls pronoms personnels, «il» est en fait impersonnel), on peut penser l’animisme comme une généralisation de la nature déictique de la position de sujet, incluant les non-humains. À partir du moment où l'on dit «tu »à un animal, on le pense nécessairement comme pouvant dire «je» :

Ce n'est donc pas tellement que les animaux, par exemple, soient des sujets parce qu'ils sont «au fond» des humains; c'est plutôt que les animaux sont conçus comme étant des humains parce qu'ils sont, d'abord, pensables comme étant des sujets. L’animisme, c'est-à-dire, l'attribution aux non-humains d'une «âme » ou intentionnalité consciente, ne dépendrait donc pas d'une projection de propriétés substantives des humains sur les non-humains, mais plutôt d'une reconnaissance des rapports réflexifs que tout être entretient avec soi-même (ibid.).

En d'autres termes, faut-il ou non prendre au sérieux le perspectivisme des Amérindiens? Pour Viveiros de Castro, Descola conserve la conviction que le seul monde subjectif réel est celui des hommes, et que les Amérindiens «attribuent» des qualités humaines aux animaux, alors que dire que les animaux sont des «gens» implique que ceux-ci peuvent inverser le «je» et le «tu», et s'assumer comme sujet réflexif. 
Quelle est la réponse de Descola? On peut la lire dans une section de Par-delà nature et culture intitulée «Animisme et perspectivisme» (2005, p. 196-202). L'animisme «standard» n’est pas nécessairement perspectiviste. Le perspectivisme considère que les animaux (qui se voient comme des humains) voient les humains comme des animaux, non comme des hommes. Or, il s'agirait pour Descola seulement d'un cas particulier d'animisme assez rare, car dans la majorité des cas, les hommes pensent que les animaux (qui sont des sujets pensant) les voient bien comme des hommes, et non comme des bêtes. Plus précisément, il paraît difficile pour un homme de tenter d'échanger quoi que ce soit avec un animal, si ce dernier ne peut percevoir l'homme comme un sujet. Les animaux se voient donc comme des sujets, tout comme les hommes auxquels ils ont affaire, mais ils savent aussi que les hommes ne sont pas des jaguars ou des tapirs : Descola diffère en ce point de Viveiros de Castro. Dans l'animisme non perspectiviste, les animaux ont connaissance du mode de vie des hommes, et ont conscience que ceux-ci peuvent être leurs ennemis, tout comme certaines tribus humaines peuvent être hostiles entre elles, parler des langues différentes et même avoir une apparence physique différente du fait des parures et des tatouages. Pour Descola, le perspectivisme introduit peut-être dans l'animisme un degré de complexité excessif que peu de tribus ont fait leur : l'animisme reste donc le plus souvent non symétrique, les hommes pensent que les animaux les voient comme les humains qu'ils sont, et non sous une forme animale.

Le débat rebondit en 2009, lorsqu'un débat est organisé entre les deux anthropologues par l'institut d'études avancées de Paris 6 . Bruno Latour en a fait un compte rendu tout à fait éclairant (Latour, 2009). Ce sociologue, partisan d'une réforme en profondeur de la sociologie contemporaine, est un interlocuteur privilégié des deux débatteurs , il n’est donc pas étonnant qu’il ait été présent lors de cet événement, qui prend la forme d’une «dispute» comme la scholastique médiévale en connut beaucoup. Descola commence par reconnaître sa dette à l'égard de l'anthropologue brésilien en ce que ce dernier l'a aidé

6 Débat organisé à la maison Suger à Paris, le 30 janvier 2009.

7 Bruno Latour est commenté par Viveiros de Castro à propos de l'«amodernité» (1996, p. 125), et chaleureusement remercié par Descola (2005, p. 553). 
à dépasser l'opposition entre nature et culture par ses travaux américanistes, et à construire les concepts d'animisme et de naturalisme. L'anthropologie peut-elle s'en tenir au contraste entre la première cosmovision, dans laquelle le monde est entièrement peuplé de sujets (animaux, humains, esprits) ayant une apparence physique variable, et la deuxième vision du monde où la nature est conçue comme homogène à tous les existants et la culture à la fois hétérogène et réservée au humains? Ces deux visions du monde sont-elles suffisantes à décrire les mœurs de toute l'humanité? La réponse de Descola est négative: il faut construire d'autres types anthropologiques pour comprendre, entre autres, les Africains ou les Australiens, dont la cosmovision n'est ni animiste ni naturaliste. Ce n'est pas le naturalisme qui est universel, mais le modèle de quatre cosmovisions, construit logiquement par une combinatoire extrêmement simple : l'intériorité des êtres peut être identiques ou distinctes, tout comme leur physicalité (2005, p. 176). Lorsque l'intériorité (la subjectivité) et la physicalité (la matérialité) sont conçues comme identiques, on peut parler de totémisme; lorsqu'elles sont pensées comme hétérogènes, on a affaire à l'analogisme. Animisme, naturalisme, totémisme et analogisme constituent donc un modèle ontologique universel, valable pour l'humanité tout entière, quelle que soit l'époque et le lieu. Le perspectivisme, comme sous-catégorie de l'animisme, n’est qu'un cas particulier d'un modèle général des relations entre humains et non-humains.

Dans la suite du débat, Viveiros de Castro conteste vivement cette relégation de son modèle au statut de type secondaire d'une classification totalisante. Le perspectivisme est totalement irréductible à une case dans une typologie. C'est une façon de penser incompatible avec une grille fixe de concepts fondamentaux telle que la propose Descola. Ce dernier aurait construit une sorte de cabinet de curiosités bien ordonné, où le perspectivisme amérindien occuperait une petite niche, au milieu d'autres cases. Voilà pour Viveiros de Castro un exemple d’anthropologie «républicaine », et même si Latour ne donne pas de précision sur le sens précis de cet anathème pour Viveiros de Castro, on peut supposer qu'il est synonyme de «pensée impérialiste» dans le cadre de ce débat. Retournant sa typologie contre Descola, l'anthropologue brésilien considère que la pensée de ce dernier est «analogique » : il accumule et classe 
une multitude d'éléments variés pour échapper à la rhapsodie de la diversité. En fin de compte, la typologie de Descola a pour effet d'atténuer la puissance subversive pour la pensée occidentale («naturaliste») du perspectivisme amérindien. Le perspectivisme est une «bombe» qui fait exploser les cadres de la pensée occidentale, et non un «type» ou une «sous-catégorie» d'un vaste système rationnel. L'entreprise de modélisation ontologique de Descola conduit, consciemment ou non, à désamorcer l'«explosif» perspectiviste. Or, si l'on adopte la pensée perspectiviste, la construction de quatre ontologies n’a absolument aucun sens. Latour rapporte que Descola déclara qu'il n’était pas intéressé par la pensée occidentale, mais par celle des autres, Viveiros de Castro lui opposant que c'était précisément sa façon d'être «intéressé » par les autres qui posait problème. À une question venant du public, Viveiros de castro répondit que «l'anthropologie aujourd'hui est largement décolonisé, mais sa théorie n'est pas encore assez décolonisée » (Latour, 2009, p. 2). Nul doute que cette réponse visait aussi Descola.

Si l'anthropologue brésilien reproche à Descola une sorte d’arrogance rationaliste, typiquement occidentale selon lui, qui vise à enfermer les cosmovisions dans des «boîtes» sur des étagères, c'est autrement que la théorie des ontologies a été lue et critiquée en France. Alors que Viveiros de Castro décrit l'ouvrage de Descola comme un exemple supplémentaire de l'hégémonie taxinomique européenne, les lectures françaises s'inscrivent dans certaines traditions scientifiques, dans des filiations épistémologiques. Comment s'est donc développé le débat français?

\section{LECTURES FRANÇAISES}

On peut identifier trois points de vue distincts sur le «descolisme » après la publication de Par-delà nature et culture, produits des orientations majeures de l'anthropologie française depuis une cinquantaine d’années. La première lecture se situe du côté de l'anthropologie d'inspiration positiviste et matérialiste, la deuxième relève de l'anthropologie dynamique dont le père fondateur en France est Georges Balandier, et enfin, la troisième est structuraliste, dans le sillage de Claude Lévi-Strauss. 
Publié environ un an après Par-delà nature et culture, la note critique de Jean-Pierre Digard (2006) s'interroge sur la confusion possible entre le constat qu'il y a plusieurs visions du monde dans différents groupes humains, aujourd'hui et par le passé, avec l'idée qu'elles se vaudraient toutes du point de vue de leur adéquation à la réalité. Après un résumé très fidèle et bienveillant de l'ouvrage, Digard émet des doutes sur la validité des thèses descolistes, «au risque de passer pour un positiviste attardé ou pour le tenant d’un humanisme à bout de souffle» (ibid., p. 422). En premier lieu, l'ouvrage pêche par une excessive sophistication et par une trop grande abstraction. Ainsi, après avoir construit une typologie quadripartite des modes d'identification (les ontologies citées ci-dessus), Descola s'emploie à définir des modes relationnels, cette fois en six types. Les trois premiers (échange, don, prédation) mettent en jeu des existants de même niveau ontologique; les trois suivants (production, protection, transmission) concernent les relations entre existants de natures différentes. Or, Digard relève que Descola reconnaît assez vite que le lien entre les deux typologies est lâche : on ne sait trop pourquoi un collectif privilégie tel ou tel mode relationnel. Pourquoi alors consacrer de longues pages à une question sans réponse? Ou plutôt, c'est encore l’animisme qui sert à Descola de modèle privilégié : la prédation est le mode relationnel animiste par excellence. Mais quid des autres ontologies du point de vue relationnel?

En deuxième lieu, Digard reste sceptique sur la capacité du modèle quadripartite à rendre compte de toutes les idéologies humaines. Descola a une connaissance de première main de la cosmovision des Achuars, et sans aucun doute de celles des Amérindiens, mais on se demande si les exemples choisis pour illustrer les autres ontologies ne sont pas ad hoc, ou pire, distordus pour les faire entrer dans le modèle. La théorie des ontologies n'est-elle pas une chambre à coucher meublées de quatre lits de Procuste? Si aucun des lits n’est à votre taille, Descola vous coupe les pieds ou vous allonge le corps! $!^{8}$ Pour Digard, l'Occident ne peut pas se réduire au naturalisme, ni à aucune des trois autres ontologies. C'est ce qui conduit Descola à caricaturer l'esprit moderne européen, en sous estimant la variabilité historique et synchronique des représentations de la nature. 
En troisième lieu, Digard (et c'est assurément en cela qu'il est positiviste) reproche à Descola de sembler croire, comme les Amérindiens, que les animaux sont des sujets comme les hommes, et que les animaux peuvent déposer leurs oripeaux et prendre forme humaine, tout comme les chamans peuvent se métamorphoser en animal et frayer avec les jaguars et les tapirs. Or, ce n'est pas parce que les Amérindiens ont de telles idées que ces idées sont vraies. Toutes les représentations du réel n'ont pas la même valeur de vérité. Contrairement à ce quavance Latour, la science n'est pas un «mythe», et l'animisme défini par Descola n'est pas une science du réel. Car fondamentalement, la question des représentations est secondaire pour Digard:

La matérialité des faits tels qu'ils sont exposés par Descola lui-même montre que les Achuars, quelle que soit l'humanité qu'ils prêtent aux animaux, chassent, tuent et mangent certains de ceux-ci, en apprivoisent et en élèvent d'autres, exerçant sur eux des actions qui ne diffèrent guère de celles qu'exercent ailleurs d'autres hommes, que par les discours et les représentations grâce auxquels ils donnent sens et cohérence à ces actions (ibid., p. 425).

Digard situe son différend dans la genèse de deux écoles anthropologiques françaises: ceux qui ont suivi Lévi-Strauss, qui se focalisent sur les représentations, et ceux qui s'inspirent de Leroi-Gourhan, en privilégiant l'étude des pratiques. Les premiers sont plutôt du côté de la philosophie (d’où la réappropriation de concepts comme celui d'ontologie), et les seconds du côté des sciences naturelles et physiques. Digard reproche ainsi à Descola une forme d'idéalisme, car il néglige de se pencher sur les conditions matérielles de la vie humaine, et sur les pratiques qui en découlent. Finalement, ce lecteur (très) critique souligne l'ambivalence, sinon la complaisance de Descola, à l'égard d'idéologies prônant la «libération animale» ou un «nouveau contrat naturel», qui peuvent entretenir une affinité avec l’anthropologie des «existants» (incluant donc les non-humains : végétaux, animaux, machines, etc.), que Descola appelle de ses vœux, et qui doit se substituer l'anthropologie «classique», c'est-à-dire à l'étude de l'homme en société. 
La réponse de Descola à cette charge «positiviste» est publiée dans le même numéro de L'Homme (Descola, 2006). À la critique d'encyclopédisme mal maîtrisé, il répond que son projet part d'hypothèses relativement abstraites et qu'«il n'est peut-être qu’une expérience de pensée ». Les exemples choisis pour illustrer les quatre ontologies ne visent en rien l'exhaustivité, ce qui serait «un projet délirant». Contrairement aux grands comparatistes comme Frazer ou Radcliffe-Brown, Descola ne procède pas par induction mais par hypothèse et déduction. Quant aux quatre ontologies, on ne peut leur reprocher d'être trop abstraites, car elles ont été définies avec une compréhension (au sens logique) suffisamment pauvre pour inclure un large périmètre (il y a de la place pour tout le monde dans ce nouveau lit de Procuste...9) : le naturalisme permet d'inclure la vision du monde du marchand de cochons, du biologiste et du contremaître, tous Européens; ce qui semble hétérogène apparaît au contraire homogène, selon Descola, du point de vue des autres ontologies. Le naturalisme européen se laisse donc voir plus aisément à partir de ce qui n'est pas lui-même. Autre objection de Digard auquel il faut répondre: Descola croit-il vraiment que les Jaguars se transforment en hommes et vice-versa? La réponse est négative (ce qui peut rassurer les mécréants ${ }^{10}$ ). En revanche, Descola affirme que les représentations sont réelles quand elles sont stables et collectives. Les porteurs de ces représentations, même si elles sont contre-intuitives, les tiennent pour vraies, et non pour des fables, des jeux de mots ou des métaphores. Ces représentations ont des effets pratiques, on ne peut donc pas les écarter d'un revers de main, arguant qu'elles sont «irréelles». Descola considère d'ailleurs que la distinction de Digard entre représentation et pratique est inopérante, les représentations sans pratique étant rares, et l'inverse impossible. Enfin, loin d'être relativiste, Descola se veut au contraire «universaliste » et reconnaît la valeur des sciences positives, en particulier de la psychologie cognitive, qui doit l'aider à fonder la genèse cérébrale des quatre ontologies. 
Si, d'une certaine façon, Viveiros de Castro et Digard prennent Descola en étau, l'un lui reprochant d'être un rationaliste impérialiste, l'autre d'être un relativiste irrationaliste, la critique de Jean-Paul Colleyn (2006) est d'une autre nature. Ce dernier note d’abord l'hétérogénéité des sources utilisées. Comment ont-été produites les données utilisées par Descola, en dehors de celles qu’il a collectées lui-même? Dans le domaine africain, dont Colleyn est un grand spécialiste, on ne peut toujours tenir pour authentique les récits de certains anthropologues, dont on sait qu'ils ont co-produit leurs informations avec quelques informateurs privilégiés seulement (c'est le cas d'une bonne partie de l'école griaulienne). Plus de prudence avec les sources secondaires aurait été bienvenue, car si la base empirique de la démonstration n’est pas fiable, quelle valeur peut-on lui accorder? Après ce point de méthode, non négligeable, la lecture de Colleyn expose deux séries d'interrogations. D’abord, les ontologies ressemblent beaucoup aux systèmes symboliques de Lévi-Strauss, alors que Descola, sans en donner la raison, n'emploie plus les mots «symbole» ni «symbolique», qui ont fait pourtant les belles heures de l'anthropologie structurale. Comme les systèmes symboliques, pourtant, les ontologies laissent perplexe quant à savoir si telle ou telle représentation ou pratique particulière, telle production culturelle, entre ou non dans l'un des quatre types. En quoi, par exemple, la sorcellerie chez les Nuers, telle que la décrit Evans-Pritchard, seraitelle plus analogique, quanimiste, totémique ou naturaliste? C'est seulement par une pétition de principe, en définissant les sociétés africaines comme typiquement analogique que l'on peut subsumer telle ou telle coutume, correspondants à l'ontologie analogique, qui en retour sont supposées attester de son existence.

Ensuite, Colleyn pose la question de la diachronie dans l'ouvrage de Descola, qui semble plus voyager dans l'espace que dans le temps. Comment passe-t-on d'une ontologie à une autre? Le chapitre 15, intitulé «Histoires de structures » traite ainsi du passage d'une société de chasseurs-cueilleurs à celui d'éleveurs, en prenant comme exemple privilégié la chasse ou bien l'élevage du caribou en Amérique du nord et du renne en Sibérie (c'est la même espèce). Si le passage d'un modèle à un autre est possible, c'est parce qu'aucune ontologie n'est absolument dominante; une ou plusieurs autres sont toujours plus ou 
moins latente, ce sont des virtualités. Dans certaines conditions, en particulier l'émergence d'un type de relation appelant un nouveau type d'identification, le passage s'effectue d'une ontologie à une autre. C'est ce qui s'est passé en Europe, selon Descola, entre le XVII et XVIII ${ }^{\mathrm{e}}$ siècle, avec la transformation de l'analogisme en naturalisme: c'est l'épiphanie de la modernité. Colleyn remarque néanmoins qu'il y certainement plusieurs genres de «modernités», et surtout que l’argumentaire de Descola vaut surtout pour de petites sociétés isolées, en non pour celles qui sont confrontées à la colonisation depuis des siècles :

On peut se demander si le type de sociétés et de cultures auquel s'intéresse de manière privilégiée Philippe Descola - à savoir des groupes réduits, de petite échelle et relativement isolés - ne facilite pas l'exercice, en même temps qu'il détermine la théorie. Comment procéder quand les critères de différenciation ethnique sont plus complexes et incompréhensibles hors de leur contexte historique? Que devient la notion de collectif quand le groupe est pris dans des réseaux plus globaux, comme par exemple les Bamana du Mali, en interaction depuis plus de dix siècles avec l'Islam? Et la notion de collectif est-elle pleinement pertinente pour l'ensemble des Modernes? (ibid., p. 3ı)

Colleyn retrouve ainsi l'orientation épistémologique de l'anthropologie dynamique, avec les analyses classiques de Balandier (1955) sur la situation coloniale, ainsi que celle de Marc Augé sur le sens et la fonction des systèmes symboliques africains (1979). Que peut-dire le structuralisme - ou l'anthropologie des ontologies - de sociétés qui connaissent les migrations, les guerres, la domination étrangère (ou l'asservissement des étrangers), la création de royaumes, d'empires, puis leur disparition, depuis des siècles? Ce sont des sociétés en changements culturels et sociaux constants, qui connaissent un haut degré d'hétérogénéité. Pour Colleyn, la pensée de Descola reste culturaliste : une ethnie, une langue, un territoire... une ontologie. Cela ne correspond en rien à la plupart des systèmes symboliques africains, ouverts depuis longtemps à la différence religieuse, linguistique et politique. Il n’est en rien étonnant, précisément, que cette objection vienne d'un africaniste reconnu : l'anthropologie africaniste, en dehors de quelques exceptions, comme les travaux de Luc De Heusch (197I) sur les Bantu, se prête mal au structuralisme. 
La troisième lecture de l'ouvrage de Descola" ${ }^{11}$ est beaucoup moins sceptique que les précédentes. Elle est au contraire extrêmement admirative. Pour Pottier, l'ouvrage est un «chef d'œuvre», «une synthèse éblouissante qui témoigne à la fois de l'érudition impressionnante de son auteur et d'une imagination théorique peu commune» (2007, p. 79I). On ne s'étonnera pas qu'elle vienne d'un chercheur dont les travaux doivent beaucoup au structuralisme. En effet, Richard Pottier, spécialiste du Laos et plus généralement des peuples de la péninsule indochinoise, est l'auteur d'une remarquable étude des mythes de la déesse du riz (1994), dont la base théorique vient largement de Lévi-Strauss et Greimas. Quels sont donc pour lui les mérites de cette nouvelle anthropologie des ontologies?

D’abord, contrairement à Lévi-Strauss, il cherche des invariants non sous la forme de structures mentales, ce qui relève de la psychologie ou des neurosciences, mais sous celle de schèmes intermédiaires de type identificatoire (les quatre ontologies) ou relationnelle (les six modes de relation). Ces schèmes reflètent les dispositions humaines innées et les contraintes de la vie sociale, et se manifestent («se concrétisent» écrit Pottier) dans les pratiques et les représentations observables. En outre, Pottier défend vigoureusement la scientificité de la démarche de Descola, contre l'attaque de Digard. Selon Pottier, il est ainsi légitime à la fois de relativiser le naturalisme, en la considérant tout aussi «arbitraire » que les quatre autres, et en même temps de considérer que cette cosmogonie a permis l'essor de la pensée scientifique. Je ne suis pas certain que Descola considère les ontologies comme «arbitraires »; au contraire, il tend à les considérer - en tant que système quaternaire - comme universelle, c'est-àdire comme nécessaires. Mais le problème reste, quoi qu'en pense Pottier, entier : soit l'ontologie naturaliste est la seule vraie, ce qui signifie qu'elle est la seule qui soit ontologiquement fondée, les autres étant alors des variations imaginaires, sans rapport avec la réalité, soit c'est le système des quatre ontologies qui est

II La Revue française de sociologie, dont les éditeurs étaient conscients de l'importance de Pardelà nature et culture, demandèrent à un anthropologue (Richard Pottier) et un sociologue (François Héran) de donner leur avis sur l'ouvrage. Je m'en tiendrai à une présentation de la première recension, afin de rester dans le champ disciplinaire de l'ethnologie (la réception de l'œuvre de Descola hors discipline, en sociologie ou en histoire, nous conduirait trop loin). 
vrai, aucune des positions n'ayant de valeur de vérité par rapport au trois autres, et le naturalisme n'est pas plus vrai que les autres dispositifs symboliques.

Pottier, qui annonçait qu'il ne ferait aucune critique contre Descola, ne peut néanmoins s'empêcher d'en faire au moins une, qui me semble parfaitement fondée, même dans un cadre structuraliste :

Le seul point qui me laisse un peu perplexe dans les analyses de Descola, c'est la définition qu'il donne de l'analogisme. Certes cette définition découle de la nécessité de remplir la quatrième case de la combinatoire qu'il propose en trouvant un système qui soit complémentaire du totémisme. Un tel argument n'est cependant pas convaincant, car l'on sait bien qu'une combinatoire peut parfaitement comporter une case vide. On ne saurait donc se passer d'une confirmation empirique. La question est d’autant plus cruciale que, par rapport aux trois autres cas de figure envisagés, l’analogisme est surreprésenté, puisqu'il concerne toutes les civilisations historiques de l’Ancien et du Nouveau monde (2007, p. 792).

En d'autres termes, l'analogisme est une fausse fenêtre, beaucoup trop large, dans le système de Descola. Par esprit de symétrie, l’anthropologue s'est employé à trouver des exemples d'analogies qui ne correspondent pas à une double discontinuité des intériorités et des physicalités. Pottier remarque avec justesse que l'analogie est une forme de pensée qui assimile des phénomènes à des messages. Le passage de l'analogisme au naturalisme est alors, plus simplement que le pense Descola, le passage d'un monde qui fait sens, fourmillant de significations qu'il faut savoir lire, à un monde objectivable et analysable en termes lois de la nature, de causes et d'effets. Pour Pottier, ce passage est irréversible, et jamais le monde ne sera réenchanté. En revanche, le dépassement du naturalisme peut se faire sur un plan éthique, l’homme devant apprendre à préserver le monde où il vit, et dont il fait partie. Détruire la «nature», c'est se détruire soi-même. C'est en cela que le naturalisme conquérant peut être dépassé.

Ces trois points de vue typiquement français, compréhensibles si l'on prend en compte l'histoire nationale de l'anthropologie, doivent maintenant être mis en perspective par la réception nord-américaine, qui prend place dans un contexte disciplinaire différent. 


\section{AUX ÉTATS-UNIS}

L’anthropologie américaine a une histoire spécifique, assez distincte de celle du Royaume-Uni et de celle de la France (Dianteill, 20I2). Si la discipline dans ces deux derniers pays a connu une forte influence durkheimienne, puis fonctionnaliste et structuraliste, l'anthropologie nord-américaine s'est constituée d'abord comme culturelle, dans le sillage de l'école de Franz Boas, puis s'est diversifiée dans les années 1960, avant de connaître un tournant post-moderne à la fin des années 1980, en mettant en doute les vastes généralisations de type structuraliste ou fonctionnaliste, pour se concentrer sur des études de cas et sur l'épistémologie critique de la discipline.

La préface de Marshall Sahlins, professeur à l'université de Chicago, avec l'ironie qui le caractérise, prévient lecteur nord-américain que l'ouvrage de Descola risque de le surprendre. Celui-ci est en effet habitué à une rhapsodie d'études ethnographiques sur des sujet excessivement précis (l'énumération effectuée par Sahlins fait sourire par l'incongruité des études de terrain publiées ces dernières années dans les revues d’anthropologie aux États-Unis : les douanes au Ghana, l'élevage des porcs en Caroline du Nord, les femmes israéliennes dans la guerre du Liban en 2006, la psychiatrie grecque, etc.) conduisant à une fragmentation extrême du champ des études anthropologiques. Pour Sahlins, le seul principe unificateur ayant survécu aux États-Unis à la «Terreur » déconstructiviste et anti-structuraliste est une explication généralisée par la globalisation du capitalisme (2013, p. xii). Or, l'ouvrage de Descola propose justement un changement radical d'orientation pour la discipline, un changement de paradigme opposé à l'aversion contemporaine pour la théorie générale en anthropologie. Plus loin, Sahlins souligne que les propositions de Descola forment une sorte de révolution copernicienne: l'anthropologie occidentale ne doit plus faire tourner les mondes des autres peuples autour du sien (en les pensant dans un cadre naturaliste), mais au contraire, tourner autour d'eux pour relativiser les catégories qui la soutiennent, en particulier l'opposition Nature/Culture. Ainsi, la notion de «production», chère aux marxistes, n’a pas d'universalité : les chasseurs-cueilleurs, imprégnés d'une ontologie animiste, ne conçoivent pas l’activité cynégétique ainsi, mais comme 
don, prédation ou échange avec les animaux et les végétaux, dont ils pensent qu'ils ont une âme. Pour Sahlins, enfin, l'ouvrage de Descola n'est peut-être pas la dernière des grandes tentatives comparatives (on suppose que Sahlins pense à Morgan, Frazer ou Lévi-Strauss): «Bien que le vaste schéma comparatif du Professeur Descola, sur le modèle des grands classiques (old-timers), puisse paraître à certains être l'oiseau de Minerve prenant son vol au crépuscule, on peut arguer avec force que c'est plutôt Chanteclair, le coq gaulois ${ }^{12}$, annonçant une nouvelle aube anthropologique» (ibid., p.xiv, traduit par nous).

Un parrainage aussi prestigieux ne pouvait passer inaperçu, et c'est probablement l'une des raisons pour laquelle la revue en ligne HAU: Journal of Ethnographic Theory (20I4, 4-3) a consacré un symposium à Beyond Nature and Culture. Le dossier comprend sept notes critiques, et une réponse de Descola. Je présenterai seulement les deux textes écrits par les chercheurs nord-américains, moins par souci d'économie que parce qu'ils me semblent aborder des points tout à fait typiques des préoccupations anthropologiques outre-Atlantique.

Stefan Helmreich, professeur d'anthropologie des sciences au Massachussetts Institute of Technology, remarque que la question du genre est presque totalement absente de l'ouvrage de Descola. Le mot gender n'apparaît qu'une seule fois dans la version anglaise. Selon Helmreich, il existe bien un riche corpus de réflexions sur la question des rapports entre le couple Nature/Culture et la différence sexuelle, mais ce sont des philosophes qui ont réfléchi sur ce sujet: Simone de Beauvoir, Luce Irigaray, Hélène Cixous, Julia Kristeva, par exemple. En revanche, l’anthropologie française, selon lui, s'est peu intéressée à la politique des sexes (ou du genre), notamment dans les sociétés industrielles et postcoloniales, contrairement aux Américains et aux Britanniques. Dans sa «forme canonique», l’anthropologie française serait plus préoccupée des universaux humains à trouver dans des sociétés isolées du contact avec l'Occident. Helmreich a de l'anthropologie française une vue un peu étroite, limitée au structuralisme, mais il pointe néanmoins un angle mort du travail de Descola, à savoir que la relativisation du naturalisme devrait passer 
par une critique de la naturalisation des catégories de «genre» (ou de sexe social). On pourrait faire une critique identique sur tous les thèmes, néanmoins, dans le cas présent, elle est particulièrement justifiée. Ne serait-ce pas la différence sexuelle qui serait fondamentalement à l'origine de l'opposition Nature/Culture (Ortner, 1974) ? Descola (200I) a effectivement abordé le sujet du «genre » chez les Amérindiens, soutenant que la relation entre humains et non-humains conditionnait la définition des rôles sexués chez eux, mais on se demande pourquoi la question n'est pas traitée pour l'ontologie naturaliste dans son opus magnum. Si c'est la différence sexuelle qui explique l'opposition Nature/ Culture, alors quel est le pouvoir explicatif du couple Intériorité/Physicalité (le naturalisme se caractérisant par la continuité des physicalités et la disjonction des intériorités) ? Finalement, en louant le caractère fictif et littéraire de tout l'ouvrage de Descola, la recension de Helmreich (2014) s'achève sur un point d'orgue post-moderne; il n'est pas certain que l'anthropologue français, qui reste attaché à la scientificité de l'anthropologie, ait apprécié ce compliment :

À la fin de son livre, Descola avoue qu'il est imprégné par la vision du monde naturaliste, et que par conséquent, d'une certaine manière, le texte entier a été un rêve rêvé par une ontologie. Pour moi, cette fin à la Alice au pays des merveilles est une partie à la fois élégante et révélatrice du livre, c'est le moment où nous réalisons que ce texte est de la science fiction, au plein sens du terme, une collecte et une extrapolation de faits et de phénomène de notre monde, dans le but de nous montrer comment nous pourrions l'imaginer autrement (ibid., p. 379, traduit par nous).

L’article de Michael Lambek, professeur d’anthropologie de la religion à l'université de Toronto $^{13}$, est d'une tout autre facture, dans le fond comme dans la forme. Judicieusement, il remarque que l'ouvrage de Descola s'apparente à un déplacement des méthodes des Structures élémentaires de la parenté (1949) à La Pensée sauvage (1962), le modèle restant bien évidemment le structuralisme lévistraussien. Contrairement à Marshall Sahlins, Lambek (20I4, p. 4I2) considère que l'ouvrage n'est pas un «tournant» (turn) pour la discipline, mais la résurgence et le renouveau du structuralisme après la combinatoire «sans fin» des

13 Michael Lambek est canadien, et non citoyen des États-Unis; il enseigne néanmoins dans une université anglophone nord-américaine, ce qui me conduit à présenter sa recension dans cette section, d'autant qu'il y débat brièvement avec Marshall Sahlins. 
Mythologiques (1964-197I) ayant montré ses limites. Lambek a bien conscience, néanmoins, que Descola n'est pas un épigone de Lévi-Strauss, mais qu'il a pour ambition de déconstruire l'opposition Nature/Culture en montrant son lien fort à la modernité occidentale. Lambek doute de la pertinence de cette thèse, et propose une «expansion» plutôt qu'un abandon du couple Nature/Culture (notons d’ailleurs que «physicalité» trouve son étymologie dans la physis grecque, c'est-à-dire la «nature »). Ces concepts sont nécessaires à la compréhension de ce que pensent et ce que font les gens, même s'il faut en donner une définition moins hétérogène, plus claire et plus abstraite que l'usage dans le langage courant. «Par-delà» ne doit pas signifier «contre» Nature et Culture, mais l'adoption d'une focale plus large. Pour Lambek, Descola va assurément au-delà de l'opposition en combinant une distinction substantive (Intériorité/ Physicalité) et une distinction logique (Similarité/Différence). Mais ce n’est qu'une autre façon, plus complexe, de penser la différence entre nature et culture. Tous les peuples posent la question de ce qui rapproche et distingue l'homme des non-humains, physiquement et mentalement, c'est-à-dire du point de vue de la nature et de la culture. Certes, tous ne pensent pas comme les modernes occidentaux, mais faut-il pour cela abandonner ces concepts? L'ontologie (au singulier ou au pluriel) peut-elle se substituer avec profit au couple Nature/Culture? Le gain heuristique n'est pas certain.

Enfin, Lambek souligne avec justesse que si le degré d’abstraction des quatre ontologies est, par construction, exactement le même, leur réalisation avérée dans le monde réel est profondément déséquilibrée. Le totémisme, sous la plume de Descola, semble se limiter à l'Australie et le naturalisme à l'Occident européen. L'animisme est avant tout américain et en partie asiatique, même si son importance épistémologique semble décisive dans la construction du modèle (Lambek remarque en note que c'est l'émergence sur le devant de la scène anthropologique de l'ethnographie amérindienne qui est à l'origine du prétendu «tournant» ontologique - ce quatteste le débat entre Viveiros de Castro et Descola). L’analogisme semble avoir une extension très large, de l'Afrique à l'Europe prémoderne, en passant par l'Asie. Lambek note que c'est l'un des points faibles du système ontologique: «L’analogisme, placé dans la case opposée de 
l'animisme, est peut-être le mode ontologique le moins convaincant, en partie parce qu'il est trop large et compréhensif, et parce qu'il sert de reliquat aux trois autres modes, plus clairement délimités » (2014, p.416, traduit par nous). Ce qui apparaît dans ces dernières remarques, c'est le problème de la congruence du modèle rationnel avec la réalité. Lambek rejoint ici Pottier: une typologie formelle n'implique pas nécessairement l'existence concrète.

\section{CONCLUSION}

Après dix ans de controverses ayant suivi la publication de Par-delà nature et culture, après avoir subi le stress test de la critique internationale, le modèle descolien est-il toujours robuste? Tous les lecteurs ont manifesté leur admiration pour un ouvrage monumental, qui allie un effort théorique magistral, une érudition ethnologique exceptionnelle et une ethnographie amérindienne de première main. Descola est à la fois anthropologue, ethnologue comparatiste et ethnographe: toutes les recensions saluent un tel tour de force. Mais même les lectures les plus bienveillantes, comme celle de Pottier, ne peuvent éviter de pointer les failles de l'anthropologie des ontologies. Sans reprendre l'intégralité des arguments invoqués, on peut identifier trois faisceaux critiques qui correspondent à des épistémologies concurrentes du structuralisme : le rationalisme positiviste, l'anthropologie dynamique et le post-modernisme. À titre personnel, je ferai donc finalement quatre propositions éclectiques (restons doublement binaires!), inspirées par ces positions épistémologiques.

D’abord, en mettant en question l'universalité du couple Nature/Culture, Descola inquiète les rationalistes (en particulier les matérialistes) car il semble accorder une légitimité ontologique à l'animisme : les animaux ont-ils une âme ? Voilà une question totalement incongrue pour un anthropologue marxiste, par exemple. Même lorsqu'il précise qu'il ne croit pas que les chamans se transforment en jaguar, il reste que Descola ne lève pas complètement l'ambiguïté sur la valeur de vérité de son modèle. Si le système doublement binaire des ontologies est universel (c'est ce que Descola nomme l'universalisme relatif), comment se fait-il que la science ne repose que sur l'un d'entre eux, à savoir le naturalisme? La physique nucléaire ne peut pas reposer sur une ontologie autre que 
naturaliste : les atomes n'ont pas d'intentionnalité, la nature est soumise à des lois totalement étrangères au principe de signification. Bref, pour Descola, toutes les ontologies sont d'égale valeur, mais l'une vaut plus que les autres. Le seul moyen d'échapper à cette aporie est de considérer, comme Pottier, que c'est la valeur éthique des trois ontologies non naturalistes qui équivaut à celle du naturalisme. Mais alors, on échappe à l'ontologie pour verser dans la question morale du «devoir être», non de l'être. I) Il vaudrait mieux alors parler de quatre systèmes éthiques d'égale valeur que de quatre ontologies équivalentes au regard de la vérité.

Ensuite, en posant son modèle comme système «ontologique», Descola tombe sous la critique adressée par l'anthropologie dynamique à toute pensée qui privilégie l'ordre, la logique et la stabilité. L'être se définit évidemment comme ce qui est toujours identique à lui-même. Descola est bien un lointain héritier de Parménide, alors que les dynamistes sont les descendants d’Héraclite, pour qui, comme on le sait, «tout change» et «la guerre est partout». Quel est le degré de stabilité des représentations décrites par Descola? Sont-elles même systémiques? On peut au contraire supposer que les conceptions relatives à la nature peuvent être extrêmement variables synchroniquement dans une même société, et qu’un groupe ou un individu peut changer d'avis sur l'âme des animaux, ou même n’avoir aucun avis sur la question. Ajoutons que ces avis divergents peuvent s'affronter, coexister séparément, s’atténuer jusqu’à se confondre. Descola n’utilise ainsi aucun des travaux portant sur le syncrétisme, le métissage, l'hybridation, dans la tradition de Melville Herskovits, de Roger Bastide (lignage savant dont nous sommes quelques uns à nous réclamer). Pourquoi la société et l'histoire serait-elle toujours régies par des principes logiques? On a vu plus haut la faible valeur explicative de certaines catégories forgées logiquement par Descola, qui sont peut-être de fausses fenêtres: en caricaturant, le totémisme est nulle part, et l'analogisme partout. 2) Ne faut-il pas plutôt accepter que les façons de penser la nature sont arbitraires et instables, sans logique systématique? Nous savons depuis les travaux de Georges Balandier (1988) qu'il est indispensable de porter le regard sur le désordre car le monde humain est largement aléatoire et imprévisible. C'est une hypothèse peu satisfaisante pour un esprit formaliste, mais elle correspond certainement mieux à la réalité sociale que l'identification du rationnel et du réel. 
Enfin, même si elle a bien mauvaise presse en France, on ne peut ignorer les thématiques abordées par l'anthropologie post-moderne. En nous inspirant des Gender Studies, posons la question suivante: 3) La construction sociale des identités sexuelles, qui repose sur la naturalisation des rôles masculins et féminins, est-elle compatible avec une analyse en termes d'ontologies? Les catégories de «genre» sont des concepts politiques, en ce qu'elles sont déséquilibrées, ce qui permet d'établir une hiérarchie et une inégalité de pouvoir fondée en «nature » : la réponse est donc négative. De façon étonnante, les travaux de Françoise Héritier (qui ne sont pas postmodernes, mais se situent dans le sillage de Lévi-Strauss) sur la valence différentielle des sexes sont absents des réflexions de Descola, alors que la différence sexuelle y apparaît comme construite socialement et historiquement, même si elle est aussi fondée en «nature», autrement dit dans la vie organique. Enfin, et c'est une orientation que les post-modernes ont peut-être suivi jusqu'à l'absurde, les ontologies s'expriment nécessairement dans un langage. Faut-il considérer toute la vie sociale comme un texte? Peut-être pas, mais il reste que les ontologies s'expriment sous forme de genres oraux ou écrits (poésie, narration de mythes, forme de la conversation), souvent entre fiction et réalité : comment trancher entre cosmovision «sérieuse» et jeu de langage métaphorique? 4) Il faut, avant de statuer sur le «réalisme» des cosmovisions, mesurer leur degré de «littérarité». Terminons ainsi sur un bel exemple de textualisme multi-ontologique. Charles Perrault, auteur des célèbres contes de fées «animistes » (Le Petit Chaperon rouge et le Chat botté, par exemple, où les animaux sont des «gens»), se déclare aussi fièrement un Moderne. En témoigne le texte qui suit, où l'on notera le dualisme entre l'esprit et le corps tout comme l'universalité de la nature, typiques du «naturalisme» :

À former les esprits comme à former les corps,

La nature en tout temps fait les mêmes efforts;

Son être est immuable, et cette force aisée

Dont elle produit tout ne s'est point épuisée :

Jamais l'astre du jour qu'aujourd'hui nous voyons

N'eut le front couronné de plus brillants rayons;

Jamais dans le printemps les roses empourprées 
D’un plus vif incarnat ne furent colorées.

Non moins blanc quautrefois brille dans nos jardins

L'éblouissant émail des lis et des jasmins...

(Perrault, I842, p. 333-334).

\section{BIBLIOGRAPHIE}

AUGÉ Marc, 1979, Symbole, Fonction, Histoire, Paris, Hachette.

BALANDIER Georges, 1955, Sociologie actuelle de l'Afrique Noire, Paris, PUF. -, 1988, Le Désordre. Éloge du mouvement, Paris, Fayard.

COLLEYN Jean-Paul, 2006, «De la manière d'habiter le monde», Critique, 707, p. 302-310.

CORAZZON Raul, 201I, «Birth of a New Science: the History of Ontology From Suarez to Kant», in «Theory and history of ontology», site Internet: <http://www.ontology.co/history.htm>.

DE Heusch Luc, 197I, Pourquoi l'épouser? et autres essais, Paris, Gallimard, «Bibliothèque des sciences humaines».

DESCOLA Philippe, 1986, La Nature domestique. Symbolisme et praxis dans l'écologie des Achuar, Éditions de la Maison des Sciences de l'Homme, Paris.

-, 200I, «The Genres of Gender: Local Models and Global Paradigms in the Comparison of Amazonia and Melanesia », in Gender in Amazonia and Melanesia: an exploration of the comparative method, Thomas Gregor, Donald Tuzin (dir.), Berkeley, University of California Press, p. 91-114.

-, 2005, Par-delà nature et culture, Paris, Gallimard, «Bibliothèque des sciences humaines 》.

-, 2006, «Soyez réalistes, demandez l'impossible», L'Homme, I77-178, p. 429-434.

-, 2013, Beyond nature and culture, Chicago, The University of Chicago Press.

DIANTEILL Erwan, 2012, «Anthropologie culturelle ou anthropologie sociale? Une dispute transatlantique», L'Année sociologique, 62-I, p. 93-122. 
DIGARD Jean-Pierre, 2006, Canards sauvages ou enfants du Bon Dieu?, Paris, Éditions de l'EHESS.

HELMREICH Stefan, 2014, «The Left Hand of Nature and Culture», HAU: Journal of Ethnographic Theory, 4-3, p. 373: <doi: http://dx.doi.org/l0.143/8/hau4.3.024>.

INGOLD Tim, 1991, «Becoming Persons: Consciousness and Sociality in Human Evolution», Cultural Dynamics, IV-3, p. 355-378.

LAMBEK Michael, 2014, «The Elementary Structures of Being (Human)», HAU: Journal of Ethnographic Theory, 4-3, p.4II : <doi: http://dx.doi.org/l0.14318/hau4.3.028>.

LATOUR Bruno, 2009, «Perspectivism: "Type" or "Bomb"? », Anthropology today, 25 , p. $1-2$.

LÉVI-STRAUSS Claude, 1949, Les Structures élémentaires de la parenté, Paris, PUF.

-, 1962, La Pensée sauvage, Paris, Plon.

-, 1964-1971, Mythologiques, Paris, Plon.

ORTNER Sherry B., 1974, «Is Female to Male as Nature is to Culture?», Feminist studies, I, p. 5-31.

PERRAULT Charles, 1842, Mémoires, contes et autres œuvres de Charles Perrault: précédés d'une notice sur l'auteur, Paris, C. Gosselin, 380 p.

POTTIER Richard, 1994, Essai d'anthropologie du mythe, Paris, Éd. Kimé, «Le sens de l'histoire».

-, 2007, «Dépasser le naturalisme: pour un nouvel humanisme», Revue française de sociologie, 48-4, p. 781-793.

SAHLINS Marshall, 2013, «Foreword», in Beyond nature and culture, Philippe Descola (dir.), Chicago, University of Chicago Press, p.xi-xiv.

VIVEIROS DE CASTRO Eduardo, 1996, «Os pronomes cosmólogicos e o perspectivismo ameríndio », Mana, 2-2, p. I15-144.

-, 1998, «Conférence de M. Eduardo Viveiros de Castro», Annuaire de l'EPHE, section des sciences religieuses, 107, p. 105-108. 